\title{
EXISTENCE OF SOLUTIONS FOR ELLIPTIC EQUATIONS HAVING NATURAL GROWTH TERMS IN ORLICZ SPACES
}

\author{
A. ELMAHI AND D. MESKINE
}

Received 24 September 2003

Existence result for strongly nonlinear elliptic equation with a natural growth condition on the nonlinearity is proved.

\section{Introduction}

Let $\Omega$ be a bounded domain in $\mathbb{R}^{N}(N \geq 2)$ with the segment property.

Consider the nonlinear Dirichlet problem

$$
A(u)+g(x, u, \nabla u)=f
$$

where $A(u)=-\operatorname{div} a(x, u, \nabla u)$ is a Leray-Lions operator defined on $D(A) \subset W_{0}^{1} L_{M}(\Omega) \rightarrow$ $W^{-1} L_{\bar{M}}(\Omega)$ with $M$ an $N$-function and where $g$ is a nonlinearity with the "natural" growth condition

$$
|g(x, s, \xi)| \leq b(|s|)(c(x)+M(|\xi|))
$$

and which satisfies the classical sign condition $g(x, s, \xi) s \geq 0$. The right-hand side $f$ is assumed to belong to $W^{-1} E_{\bar{M}}(\Omega)$.

It is well known that Gossez [12] solved (1.1) in the case where $g$ depends only on $x$ and $u$. If $g$ depends also on $\nabla u$, existence theorems have recently been proved by Benkirane and Elmahi in $[3,4]$ by making some restrictions.

In [3], $g$ is supposed to satisfy a "nonnatural" growth condition of the form

$$
|g(x, s, \xi)| \leq b(|s|)(c(x)+P(|\xi|)) \quad \text { with } P \ll M \text {, }
$$

and in [4], $g$ is supposed to satisfy a natural growth of the form (1.2) but the result is restricted to $N$-functions $M$ satisfying a $\Delta_{2}$-condition.

It is our purpose in this paper to extend the result of [4] to general $N$-functions (i.e., without assuming a $\Delta_{2}$-condition on $\left.M\right)$ and hence generalize the results of $[3,4,7]$. 
As an example of equations to which the present result can be applied, we give

(1)

$$
\begin{gathered}
-\operatorname{div}\left(\exp (m|u|) \frac{\exp (|\nabla u|)-1}{|\nabla u|^{2}} \nabla u\right)+u \sin ^{2} u \exp (|\nabla u|)=f, \quad m \geq 0, \\
\text { with } f=f_{0}+\sum_{i=1}^{N} \frac{\partial f_{i}}{\partial x_{i}}, \int_{\Omega} f_{i} \log \left|f_{i}\right| d x<\infty
\end{gathered}
$$

(2)

$$
-\operatorname{div}\left(\frac{p(|\nabla u|)}{|\nabla u|} \nabla u\right)+u g(u) p(|\nabla u|)=f
$$

with suitable data $f$, where $p$ is a given positive and continuous function which increases from 0 to $+\infty$ and where $g$ is a positive function on $\mathbb{R}$.

For classical existence results for nonlinear elliptic equations in Orlicz-Sobolev spaces, see, for example, $[2,3,4,6,8,9,10]$.

\section{Preliminaries}

2.1. Let $M: \mathbb{R}^{+} \rightarrow \mathbb{R}^{+}$be an $N$-function, that is, $M$ is continuous and convex, with $M(t)>$ 0 for $t>0, M(t) / t \rightarrow 0$ as $t \rightarrow 0$, and $M(t) / t \rightarrow \infty$ as $t \rightarrow \infty$.

Equivalently, $M$ admits the following representation: $M(t)=\int_{0}^{t} m(\tau) d \tau$, where $m$ : $\mathbb{R}^{+} \rightarrow \mathbb{R}^{+}$is nondecreasing and right continuous, with $m(0)=0, m(t)>0$ for $t>0$, and $m(t) \rightarrow \infty$ as $t \rightarrow \infty$.

The $N$-function $\bar{M}$, conjugate to $M$, is defined by $\bar{M}(t)=\int_{0}^{t} \bar{m}(\tau) d \tau$, where $\bar{m}: \mathbb{R}^{+} \rightarrow$ $\mathbb{R}^{+}$is given by $\bar{m}(t)=\sup \{s: m(s) \leq t\}($ see $[1,14,15])$.

The $N$-function $M$ is said to satisfy the $\Delta_{2}$-condition if, for some $k>0$,

$$
M(2 t) \leq k M(t) \quad \forall t \geq 0
$$

When (2.1) holds only for $t \geq$ some $t_{0}>0$, then $M$ is said to satisfy the $\Delta_{2}$-condition near infinity.

We will extend these $N$-functions into even functions on all $\mathbb{R}$.

Let $P$ and $Q$ be two $N$-functions. $P \ll Q$ means that $P$ grows essentially less rapidly than $Q$, that is, for each $\varepsilon>0$,

$$
\frac{P(t)}{Q(\varepsilon t)} \longrightarrow 0 \quad \text { as } t \longrightarrow \infty
$$

This is the case if and only if

$$
\lim _{t \rightarrow \infty} \frac{Q^{-1}(t)}{P^{-1}(t)}=0
$$

2.2. Let $\Omega$ be an open subset of $\mathbb{R}^{N}$. The Orlicz class $\mathscr{L}_{M}(\Omega)$ (resp., the Orlicz space $L_{M}(\Omega)$ ) is defined as the set of (equivalence classes of) real-valued measurable functions 
$u$ on $\Omega$ such that

$$
\int_{\Omega} M(u(x)) d x<+\infty \quad\left(\text { resp., } \int_{\Omega} M\left(\frac{u(x)}{\lambda}\right) d x<+\infty \text { for some } \lambda>0\right) .
$$

$L_{M}(\Omega)$ is a Banach space under the norm

$$
\|u\|_{M}=\inf \left\{\lambda>0: \int_{\Omega} M\left(\frac{u(x)}{\lambda}\right) d x \leq 1\right\}
$$

and $\mathscr{L}_{M}(\Omega)$ is a convex subset of $L_{M}(\Omega)$.

The closure in $L_{M}(\Omega)$ of the set of bounded measurable functions with compact support in $\bar{\Omega}$ is denoted by $E_{M}(\Omega)$.

The equality $E_{M}(\Omega)=L_{M}(\Omega)$ holds if and only if $M$ satisfies the $\Delta_{2}$-condition for all $t$ or for $t$ large according to whether $\Omega$ has infinite measure or not.

The dual of $E_{M}(\Omega)$ can be identified with $L_{\bar{M}}(\Omega)$ by means of the pairing $\int_{\Omega} u(x) v(x) d x$, and the dual norm on $L_{\bar{M}}(\Omega)$ is equivalent to $\|\cdot\|_{\bar{M}}$.

The space $L_{M}(\Omega)$ is reflexive if and only if $M$ and $\bar{M}$ satisfy the $\Delta_{2}$-condition, for all $t$ or for $t$ large, according to whether $\Omega$ has infinite measure or not.

2.3. We now turn to the Orlicz-Sobolev space. $W^{1} L_{M}(\Omega)$ (resp., $W^{1} E_{M}(\Omega)$ ) is the space of all functions $u$ such that $u$ and its distributional derivatives up to order 1 lie in $L_{M}(\Omega)$ (resp., $E_{M}(\Omega)$ ). It is a Banach space under the norm

$$
\|u\|_{1, M}=\sum_{|\alpha| \leq 1}\left\|D^{\alpha} u\right\|_{M}
$$

thus $W^{1} L_{M}(\Omega)$ and $W^{1} E_{M}(\Omega)$ can be identified with subspaces of the product of $N+$ 1 copies of $L_{M}(\Omega)$. Denoting this product by $\Pi L_{M}$, we will use the weak topologies $\sigma\left(\Pi L_{M}, \Pi E_{\bar{M}}\right)$ and $\sigma\left(\Pi L_{M}, \Pi L_{\bar{M}}\right)$.

The space $W_{0}^{1} E_{M}(\Omega)$ is defined as the (norm) closure of the Schwartz space $\mathscr{D}(\Omega)$ in $W^{1} E_{M}(\Omega)$ and the space $W_{0}^{1} L_{M}(\Omega)$ as the $\sigma\left(\Pi L_{M}, \Pi E_{\bar{M}}\right)$ closure of $\mathscr{D}(\Omega)$ in $W^{1} L_{M}(\Omega)$.

We say that $u_{n}$ converges to $u$ for the modular convergence in $W^{1} L_{M}(\Omega)$ if for some $\lambda>0$,

$$
\int_{\Omega} M\left(\frac{D^{\alpha} u_{n}-D^{\alpha} u}{\lambda}\right) d x \rightarrow 0 \quad \forall|\alpha| \leq 1
$$

this implies convergence for $\sigma\left(\Pi L_{M}, \Pi L_{\bar{M}}\right)$.

If $M$ satisfies the $\Delta_{2}$-condition on $\mathbb{R}^{+}$(near infinity only if $\Omega$ has finite measure), then modular convergence coincides with norm convergence.

2.4. Let $W^{-1} L_{\bar{M}}(\Omega)$ (resp., $W^{-1} E_{\bar{M}}(\Omega)$ ) denote the space of distributions on $\Omega$ which can be written as sums of derivatives of order less than or equal to 1 of functions in $L_{\bar{M}}(\Omega)$ (resp., $E_{\bar{M}}(\Omega)$ ). It is a Banach space under the usual quotient norm.

If the open set $\Omega$ has the segment property, then the space $\mathscr{D}(\Omega)$ is dense in $W_{0}^{1} L_{M}(\Omega)$ for the modular convergence and thus for the topology $\sigma\left(\Pi L_{M}, \Pi L_{\bar{M}}\right)$ (cf. $\left.[9,11]\right)$. Consequently, the action of a distribution $S$ in $W^{-1} L_{\bar{M}}(\Omega)$ on an element $u$ of $W_{0}^{1} L_{M}(\Omega)$ is well defined. It will be denoted by $\langle S, u\rangle$. 


\section{The main result}

Let $\Omega$ be a bounded open subset of $\mathbb{R}^{N}(N \geq 2)$ with the segment property. Let $M$ and $P$ be two $N$-functions such that $P \ll M$.

Let $A: D(A) \subset W_{0}^{1} L_{M}(\Omega) \rightarrow W^{-1} L_{\bar{M}}(\Omega)$ be a mapping (not everywhere defined) given by

$$
A(u)=-\operatorname{div} a(x, u, \nabla u)
$$

where $a: \Omega \times \mathbb{R} \times \mathbb{R}^{N} \rightarrow \mathbb{R}^{N}$ is a Carathéodory function satisfying, for a.e. $x \in \Omega$, and for all $s \in \mathbb{R}$ and all $\xi, \xi^{*} \in \mathbb{R}^{N}, \xi \neq \xi^{*}$,

$$
\begin{gathered}
|a(x, s, \xi)| \leq \beta\left[c(x)+\bar{P}^{-1} M(\gamma|s|)+\bar{M}^{-1} M(\gamma|\xi|)\right], \\
{\left[a(x, s, \xi)-a\left(x, s, \xi^{*}\right)\right]\left[\xi-\xi^{*}\right]>0} \\
\alpha M(|\xi|) \leq a(x, s, \xi) \xi
\end{gathered}
$$

where $c(x)$ belongs to $E_{\bar{M}}(\Omega), c \geq 0$, and $\alpha, \beta, \gamma>0$.

Furthermore, let $g(x, s, \xi): \Omega \times \mathbb{R} \times \mathbb{R}^{N} \rightarrow \mathbb{R}$ be a Carathéodory function such that for a.e. $x \in \Omega$ and for all $s \in \mathbb{R}, \xi \in \mathbb{R}^{N}$,

$$
\begin{gathered}
g(x, s, \xi) s \geq 0, \\
|g(x, s, \xi)| \leq b(|s|)\left(c^{\prime}(x)+M(|\xi|)\right),
\end{gathered}
$$

where $b: \mathbb{R} \rightarrow \mathbb{R}$ is a continuous and non decreasing function and $c^{\prime}(x)$ is a given nonnegative function in $L^{1}(\Omega)$. Finally, we assume that

$$
f \in W^{-1} E_{\bar{M}}(\Omega)
$$

Consider the following elliptic problem with Dirichlet boundary condition:

$$
\begin{gathered}
u \in W_{0}^{1} L_{M}(\Omega), \quad g(x, u, \nabla u) \in L^{1}(\Omega), \quad g(x, u, \nabla u) u \in L^{1}(\Omega), \\
\langle A(u), v\rangle+\int_{\Omega} g(x, u, \nabla u) v d x=\langle f, v\rangle \\
\text { for all } v \in W_{0}^{1} L_{M}(\Omega) \cap L^{\infty}(\Omega) \text { and for } v=u .
\end{gathered}
$$

We will prove the following existence theorem.

Theorem 3.1. Assume that (3.2), (3.3), (3.4), (3.5), (3.6), and (3.7) hold true. Then there exists at least one solution $u$ of (3.8). 
Remark 3.2. Note that conditions (3.4) and (3.6) can be replaced by the following ones:

$$
\begin{gathered}
\alpha M\left(\frac{|\xi|}{\lambda}\right) \leq a(x, s, \xi) \xi \\
|g(x, s, \xi)| \leq b(|s|)\left(c^{\prime}(x)+M\left(\frac{|\xi|}{\lambda^{\prime}}\right)\right),
\end{gathered}
$$

with $\lambda^{\prime} \geq \lambda>0$.

Remark 3.3. The Euler equation of the integral

$$
\int_{\Omega}\left(a(u) \int_{0}^{|\nabla u|} \frac{M(t)}{t} d t\right) d x-\langle f, u\rangle
$$

is

$$
-\sum_{i=1}^{N} \frac{\partial}{\partial x_{i}}\left(a(u) \frac{M(|\nabla u|)}{|\nabla u|^{2}} \frac{\partial u}{\partial x_{i}}\right)+a^{\prime}(u) \int_{0}^{|\nabla u|} \frac{M(t)}{t} d t=f
$$

where $a(s)$ is a smooth function satisfying $a^{\prime}(s) s \geq 0$. Note that

$$
a^{\prime}(u) \int_{0}^{|\nabla u|} \frac{M(t)}{t} d t
$$

satisfies the growth condition (3.6) and then Theorem 3.1 can be applied to Dirichlet problems related to $(3.11)$.

Proof of Theorem 3.1

Step 1 (a priori estimates). Consider the sequence of approximate problems

$$
\begin{gathered}
u_{n} \in W_{0}^{1} L_{M}(\Omega), \\
\left\langle A\left(u_{n}\right), v\right\rangle+\int_{\Omega} g_{n}\left(x, u_{n}, \nabla u_{n}\right) v d x=\langle f, v\rangle \quad \forall v \in W_{0}^{1} L_{M}(\Omega),
\end{gathered}
$$

where

$$
g_{n}(x, s, \xi)=T_{n}(g(x, s, \xi))
$$

and where for $k>0, T_{k}$ is the usual truncation at height $k$ defined by $T_{k}(s)=\max (-k$, $\min (k, s))$ for all $s \in \mathbb{R}$.

Note that $g_{n}(x, s, \xi) s \geq 0,\left|g_{n}(x, s, \xi)\right| \leq|g(x, s, \xi)|$, and $\left|g_{n}(x, s, \xi)\right| \leq n$. Since $g_{n}$ is bounded for any fixed $n>0$, there exists at least one solution $u_{n}$ of (3.13) (see [13, Propositions 1 and 5]).

Using in (3.13) the test function $u_{n}$, we get

$$
\int_{\Omega} a\left(x, u_{n}, \nabla u_{n}\right) \nabla u_{n} d x \leq\left\langle f, u_{n}\right\rangle
$$


Consequently, one has that $\left(u_{n}\right)$ is bounded in $W_{0}^{1} L_{M}(\Omega)$. By [13, Proposition 5] (see [13, Remark 8]), $\left(a\left(x, u_{n}, \nabla u_{n}\right)\right)_{n}$ is bounded in $\left(L_{\bar{M}}(\Omega)\right)^{N}$,

$$
\int_{\Omega} g_{n}\left(x, u_{n}, \nabla u_{n}\right) u_{n} d x \leq C
$$

where $C$ is a real constant which does not depend on $n$.

Passing to a subsequence, if necessary, we can assume that

$$
\begin{gathered}
u_{n} \rightarrow u \text { weakly in } W_{0}^{1} L_{M}(\Omega) \text { for } \sigma\left(\Pi L_{M}, \Pi E_{\bar{M}}\right) \text {, strongly in } E_{M}(\Omega) \text {, and a.e. in } \Omega \text {; } \\
a\left(x, u_{n}, \nabla u_{n}\right) \rightarrow h \text { and } a\left(x, T_{k}\left(u_{n}\right), \nabla T_{k}\left(u_{n}\right)\right) \rightarrow h_{k} \text { weakly in }\left(L_{\bar{M}}(\Omega)\right)^{N} \\
\text { for } \sigma\left(\Pi L_{\bar{M}}, \Pi E_{M}\right) \text { for some } h \text { and } h_{k} \in\left(L_{\bar{M}}(\Omega)\right)^{N} .
\end{gathered}
$$

Step 2 (almost everywhere convergence of the gradients). Fix $k>0$ and let $\varphi(t)=t e^{\sigma t^{2}}$, $\sigma>0$. It is well known that when $\sigma \geq(b(k) / 2 \alpha)^{2}$, one has

$$
\varphi^{\prime}(t)-\frac{b(k)}{\alpha}|\varphi(t)| \geq \frac{1}{2} \quad \forall t \in \mathbb{R}
$$

Take a sequence $\left(v_{j}\right) \subset \mathscr{D}(\Omega)$ which converges to $u$ for the modular convergence in $W_{0}^{1} L_{M}(\Omega)\left(\right.$ cf. [11]) and set $\theta_{n}^{j}=T_{k}\left(u_{n}\right)-T_{k}\left(v_{j}\right), \theta^{j}=T_{k}(u)-T_{k}\left(v_{j}\right)$, and $z_{n}^{j}=\varphi\left(\theta_{n}^{j}\right)$.

Using in (3.13) the test function $z_{n}^{j}$, we get

$$
\left\langle A\left(u_{n}\right), z_{n}^{j}\right\rangle+\int_{\Omega} g_{n}\left(x, u_{n}, \nabla u_{n}\right) z_{n}^{j} d x=\left\langle f, z_{n}^{j}\right\rangle
$$

Denote by $\varepsilon_{i}(n, j)(i=0,1,2, \ldots)$ various sequences of real numbers which tend to 0 when $n$ and $j \rightarrow \infty$, that is,

$$
\lim _{j \rightarrow \infty} \lim _{n \rightarrow \infty} \varepsilon_{i}(n, j)=0 .
$$

In view of (3.17), we have $z_{n}^{j} \rightarrow \varphi\left(\theta^{j}\right)$ weakly in $W_{0}^{1} L_{M}(\Omega)$ for $\sigma\left(\Pi L_{M}, \Pi E_{\bar{M}}\right)$ as $n \rightarrow \infty$ and then $\left\langle f, z_{n}^{j}\right\rangle \rightarrow\left\langle f, \varphi\left(\theta^{j}\right)\right\rangle$ as $n \rightarrow \infty$. Using, now, the modular convergence of $\left(v_{j}\right)$, we get $\left\langle f, \varphi\left(\theta^{j}\right)\right\rangle \rightarrow 0$ as $j \rightarrow \infty$ so that

$$
\left\langle f, z_{n}^{j}\right\rangle=\varepsilon_{0}(n, j)
$$

Since $g_{n}\left(x, u_{n}, \nabla u_{n}\right) z_{n}^{j} \geq 0$ on the subset $\left\{x \in \Omega:\left|u_{n}\right|>k\right\}$, we have

$$
\left\langle A\left(u_{n}\right), z_{n}^{j}\right\rangle+\int_{\left\{\left|u_{n}\right| \leq k\right\}} g_{n}\left(x, u_{n}, \nabla u_{n}\right) z_{n}^{j} d x \leq \varepsilon_{0}(n, j) .
$$


The first term on the left-hand side of (3.22) reads as

$$
\begin{aligned}
\left\langle A\left(u_{n}\right), z_{n}^{j}\right\rangle= & \int_{\left\{\left|u_{n}\right| \leq k\right\}} a\left(x, u_{n}, \nabla u_{n}\right)\left[\nabla T_{k}\left(u_{n}\right)-\nabla T_{k}\left(v_{j}\right)\right] \varphi^{\prime}\left(\theta_{n}^{j}\right) d x \\
& -\int_{\left\{\left|u_{n}\right|>k\right\}} a\left(x, u_{n}, \nabla u_{n}\right) \nabla T_{k}\left(v_{j}\right) \varphi^{\prime}\left(\theta_{n}^{j}\right) d x \\
= & \int_{\Omega} a\left(x, T_{k}\left(u_{n}\right), \nabla T_{k}\left(u_{n}\right)\right)\left[\nabla T_{k}\left(u_{n}\right)-\nabla T_{k}\left(v_{j}\right)\right] \varphi^{\prime}\left(\theta_{n}^{j}\right) d x \\
& -\int_{\left\{\left|u_{n}\right|>k\right\}} a\left(x, u_{n}, \nabla u_{n}\right) \nabla T_{k}\left(v_{j}\right) \varphi^{\prime}\left(\theta_{n}^{j}\right) d x
\end{aligned}
$$

and then

$$
\begin{aligned}
\left\langle A\left(u_{n}\right), z_{n}^{j}\right\rangle= & \int_{\Omega}\left[a\left(x, T_{k}\left(u_{n}\right), \nabla T_{k}\left(u_{n}\right)\right)-a\left(x, T_{k}\left(u_{n}\right), \nabla T_{k}\left(v_{j}\right) \chi_{j}^{s}\right)\right] \\
& \times\left[\nabla T_{k}\left(u_{n}\right)-\nabla T_{k}\left(v_{j}\right) \chi_{j}^{s}\right] \varphi^{\prime}\left(\theta_{n}^{j}\right) d x \\
& +\int_{\Omega} a\left(x, T_{k}\left(u_{n}\right), \nabla T_{k}\left(v_{j}\right) \chi_{j}^{s}\right)\left[\nabla T_{k}\left(u_{n}\right)-\nabla T_{k}\left(v_{j}\right) \chi_{j}^{s}\right] \varphi^{\prime}\left(\theta_{n}^{j}\right) d x \\
& -\int_{\Omega \backslash \Omega_{j}^{s}} a\left(x, T_{k}\left(u_{n}\right), \nabla T_{k}\left(u_{n}\right)\right) \nabla T_{k}\left(v_{j}\right) \varphi^{\prime}\left(\theta_{n}^{j}\right) d x \\
& -\int_{\left\{\left|u_{n}\right|>k\right\}} a\left(x, u_{n}, \nabla u_{n}\right) \nabla T_{k}\left(v_{j}\right) \varphi^{\prime}\left(\theta_{n}^{j}\right) d x,
\end{aligned}
$$

where $\chi_{j}^{s}$ denotes the characteristic function of the subset

$$
\Omega_{j}^{s}=\left\{x \in \Omega:\left|\nabla T_{k}\left(v_{j}\right)\right| \leq s\right\} .
$$

We will pass to the limit in $n$ and in $j$ for $s$ fixed in the last three terms of the right-hand side of (3.24).

Starting with the fourth term, observe that, since

$$
\left|\nabla T_{k}\left(v_{j}\right) \chi_{\left\{\left|u_{n}\right|>k\right\}} \varphi^{\prime}\left(\theta_{n}^{j}\right)\right| \leq \varphi^{\prime}(2 k)\left|\nabla T_{k}\left(v_{j}\right)\right| \leq \varphi^{\prime}(2 k)|| \nabla v_{j} \|_{\infty}=a_{j} \in \mathbb{R},
$$

we have

$$
\nabla T_{k}\left(v_{j}\right) \chi_{\left\{\left|u_{n}\right|>k\right\}} \varphi^{\prime}\left(\theta_{n}^{j}\right) \longrightarrow \nabla T_{k}\left(v_{j}\right) \chi_{\{|u| \geq k\}} \varphi^{\prime}\left(\theta^{j}\right) \text { strongly in }\left(E_{M}(\Omega)\right)^{N} \quad \text { as } n \longrightarrow \infty \text {, }
$$

and hence

$$
\int_{\left\{\left|u_{n}\right|>k\right\}} a\left(x, u_{n}, \nabla u_{n}\right) \nabla T_{k}\left(v_{j}\right) \varphi^{\prime}\left(\theta_{n}^{j}\right) d x \longrightarrow \int_{\{|u| \geq k\}} h \nabla T_{k}\left(v_{j}\right) \varphi^{\prime}\left(\theta^{j}\right) d x \quad \text { as } n \longrightarrow \infty .
$$

Observe that

$$
\left|\nabla T_{k}\left(v_{j}\right) \chi_{\{|u| \geq k\}} \varphi^{\prime}\left(\theta^{j}\right)\right| \leq \varphi^{\prime}(2 k)\left|\nabla T_{k}\left(v_{j}\right)\right| \leq \varphi^{\prime}(2 k)\left|\nabla v_{j}\right|
$$


then, by using the modular convergence of $\left|\nabla v_{j}\right|$ in $L_{M}(\Omega)$ and Vitali's theorem, we get

$$
\nabla T_{k}\left(v_{j}\right) \chi_{\{|u| \geq k\}} \varphi^{\prime}\left(\theta^{j}\right) \longrightarrow 0
$$

for the modular convergence in $\left(L_{M}(\Omega)\right)^{N}$, and thus

$$
\int_{\{|u| \geq k\}} h \nabla T_{k}\left(v_{j}\right) \varphi^{\prime}\left(\theta^{j}\right) d x \longrightarrow 0 \quad \text { as } j \longrightarrow \infty .
$$

We have then proved that

$$
\int_{\left\{\left|u_{n}\right|>k\right\}} a\left(x, u_{n}, \nabla u_{n}\right) \nabla T_{k}\left(v_{j}\right) \varphi^{\prime}\left(\theta_{n}^{j}\right) d x=\varepsilon_{1}(n, j) .
$$

The second term on the right-hand side of (3.24) tends to (by letting $n \rightarrow \infty$ )

$$
\int_{\Omega} a\left(x, T_{k}(u), \nabla T_{k}\left(v_{j}\right) \chi_{j}^{s}\right)\left[\nabla T_{k}(u)-\nabla T_{k}\left(v_{j}\right) \chi_{j}^{s}\right] \varphi^{\prime}\left(\theta^{j}\right) d x
$$

since $a\left(x, T_{k}\left(u_{n}\right), \nabla T_{k}\left(v_{j}\right) \chi_{j}^{s}\right) \varphi^{\prime}\left(\theta_{n}^{j}\right) \rightarrow a\left(x, T_{k}(u), \nabla T_{k}\left(v_{j}\right) \chi_{j}^{s}\right) \varphi^{\prime}\left(\theta^{j}\right)$ strongly in $\left(E_{\bar{M}}(\Omega)\right)^{N}$ as $n \rightarrow \infty$ by [3, Lemma 2.3], while $\nabla T_{k}\left(u_{n}\right) \rightarrow \nabla T_{k}(u)$ weakly in $\left(L_{M}(\Omega)\right)^{N}$ by (3.17).

Since $\nabla T_{k}\left(v_{j}\right) \chi_{j}^{s} \rightarrow \nabla T_{k}(u) \chi^{s}$ strongly in $\left(E_{M}(\Omega)\right)^{N}$ as $j \rightarrow \infty$, where $\chi^{s}$ denotes the characteristic function of $\Omega_{s}=\left\{x \in \Omega:\left|\nabla T_{k}(u)\right| \leq s\right\}$, it is easy to see that

$$
\int_{\Omega} a\left(x, T_{k}(u), \nabla T_{k}\left(v_{j}\right) \chi_{j}^{s}\right)\left[\nabla T_{k}(u)-\nabla T_{k}\left(v_{j}\right) \chi_{j}^{s}\right] \varphi^{\prime}\left(\theta^{j}\right) d x \longrightarrow 0 \quad \text { as } j \longrightarrow \infty,
$$

and thus

$$
\int_{\Omega} a\left(x, T_{k}\left(u_{n}\right), \nabla T_{k}\left(v_{j}\right) \chi_{j}^{s}\right)\left[\nabla T_{k}\left(u_{n}\right)-\nabla T_{k}\left(v_{j}\right) \chi_{j}^{s}\right] \varphi^{\prime}\left(\theta_{n}^{j}\right) d x=\varepsilon_{2}(n, j) .
$$

Concerning the third term on the right-hand side of (3.24), we have

$$
-\int_{\Omega \backslash \Omega_{j}^{s}} a\left(x, T_{k}\left(u_{n}\right), \nabla T_{k}\left(u_{n}\right)\right) \nabla T_{k}\left(v_{j}\right) \varphi^{\prime}\left(\theta_{n}^{j}\right) d x \longrightarrow-\int_{\Omega \backslash \Omega_{j}^{s}} h_{k} \nabla T_{k}\left(v_{j}\right) \varphi^{\prime}\left(\theta^{j}\right) d x
$$

as $n \rightarrow \infty$ by using the fact that $\nabla T_{k}\left(v_{j}\right)$ belongs to $\left(E_{M}(\Omega)\right)^{N}$.

In view of the modular convergence of $\left(\nabla v_{j}\right)$ in $\left(L_{M}(\Omega)\right)^{N}$, we have

$$
-\int_{\Omega \backslash \Omega_{j}^{s}} h_{k} \nabla T_{k}\left(v_{j}\right) \varphi^{\prime}\left(\theta^{j}\right) d x \longrightarrow-\int_{\Omega \backslash \Omega_{s}} h_{k} \nabla T_{k}(u) d x \quad \text { as } j \longrightarrow \infty
$$

and thus

$$
-\int_{\Omega \backslash \Omega_{j}^{s}} a\left(x, T_{k}\left(u_{n}\right), \nabla T_{k}\left(u_{n}\right)\right) \nabla T_{k}\left(v_{j}\right) \varphi^{\prime}\left(\theta_{n}^{j}\right) d x=\varepsilon_{3}(n, j)-\int_{\Omega \backslash \Omega_{s}} h_{k} \nabla T_{k}(u) d x .
$$


Combining now (3.32), (3.35), and (3.38), we obtain

$$
\begin{aligned}
\left\langle A\left(u_{n}\right), z_{n}^{j}\right\rangle=\int_{\Omega} & {\left[a\left(x, T_{k}\left(u_{n}\right), \nabla T_{k}\left(u_{n}\right)\right)-a\left(x, T_{k}\left(u_{n}\right), \nabla T_{k}\left(v_{j}\right) \chi_{j}^{s}\right)\right] } \\
& \times\left[\nabla T_{k}\left(u_{n}\right)-\nabla T_{k}\left(v_{j}\right) \chi_{j}^{s}\right] \varphi^{\prime}\left(\theta_{n}^{j}\right) d x-\int_{\Omega \backslash \Omega_{s}} h_{k} \nabla T_{k}(u) d x+\varepsilon_{4}(n, j) .
\end{aligned}
$$

We now turn to the second term on the left-hand side of (3.22). We have

$$
\begin{aligned}
& \left|\int_{\left\{\left|u_{n}\right| \leq k\right\}} g_{n}\left(x, u_{n}, \nabla u_{n}\right) z_{n}^{j} d x\right| \\
& \quad=\left|\int_{\left\{\left|u_{n}\right| \leq k\right\}} g_{n}\left(x, T_{k}\left(u_{n}\right), \nabla T_{k}\left(u_{n}\right)\right) z_{n}^{j} d x\right| \\
& \quad \leq \int_{\Omega} b(k) c^{\prime}(x)\left|\varphi\left(\theta_{n}^{j}\right)\right| d x+b(k) \int_{\Omega} M\left(\left|\nabla T_{k}\left(u_{n}\right)\right|\right)\left|\varphi\left(\theta_{n}^{j}\right)\right| d x \\
& \quad \leq \frac{b(k)}{\alpha} \int_{\Omega} a\left(x, T_{k}\left(u_{n}\right), \nabla T_{k}\left(u_{n}\right)\right) \nabla T_{k}\left(u_{n}\right)\left|\varphi\left(\theta_{n}^{j}\right)\right| d x+\varepsilon_{5}(n, j) .
\end{aligned}
$$

The first term of the right-hand side of this inequality reads as

$$
\begin{aligned}
\frac{b(k)}{\alpha} \int_{\Omega} & {\left[a\left(x, T_{k}\left(u_{n}\right), \nabla T_{k}\left(u_{n}\right)\right)-a\left(x, T_{k}\left(u_{n}\right), \nabla T_{k}\left(v_{j}\right) \chi_{j}^{s}\right)\right] } \\
& \times\left[\nabla T_{k}\left(u_{n}\right)-\nabla T_{k}\left(v_{j}\right) \chi_{j}^{s}\right]\left|\varphi\left(\theta_{n}^{j}\right)\right| d x \\
& +\frac{b(k)}{\alpha} \int_{\Omega} a\left(x, T_{k}\left(u_{n}\right), \nabla T_{k}\left(v_{j}\right) \chi_{j}^{s}\right)\left[\nabla T_{k}\left(u_{n}\right)-\nabla T_{k}\left(v_{j}\right) \chi_{j}^{s}\right]\left|\varphi\left(\theta_{n}^{j}\right)\right| d x \\
& -\frac{b(k)}{\alpha} \int_{\Omega} a\left(x, T_{k}\left(u_{n}\right), \nabla T_{k}\left(u_{n}\right)\right) \nabla T_{k}\left(v_{j}\right) \chi_{j}^{s}\left|\varphi\left(\theta_{n}^{j}\right)\right| d x
\end{aligned}
$$

and, as above, it is easy to see that

$$
\frac{b(k)}{\alpha} \int_{\Omega} a\left(x, T_{k}\left(u_{n}\right), \nabla T_{k}\left(v_{j}\right) \chi_{j}^{s}\right)\left[\nabla T_{k}\left(u_{n}\right)-\nabla T_{k}\left(v_{j}\right) \chi_{j}^{s}\right]\left|\varphi\left(\theta_{n}^{j}\right)\right| d x=\varepsilon_{6}(n, j)
$$

and that

$$
-\frac{b(k)}{\alpha} \int_{\Omega} a\left(x, T_{k}\left(u_{n}\right), \nabla T_{k}\left(u_{n}\right)\right) \nabla T_{k}\left(v_{j}\right) \chi_{j}^{s}\left|\varphi\left(\theta_{n}^{j}\right)\right| d x=\varepsilon_{7}(n, j)
$$

so that

$$
\begin{aligned}
\left|\int_{\left\{\left|u_{n}\right| \leq k\right\}} g_{n}\left(x, u_{n}, \nabla u_{n}\right) z_{n}^{j} d x\right| \\
\leq \frac{b(k)}{\alpha} \int_{\Omega}\left[a\left(x, T_{k}\left(u_{n}\right), \nabla T_{k}\left(u_{n}\right)\right)-a\left(x, T_{k}\left(u_{n}\right), \nabla T_{k}\left(v_{j}\right) \chi_{j}^{s}\right)\right] \\
\quad \times\left[\nabla T_{k}\left(u_{n}\right)-\nabla T_{k}\left(v_{j}\right) \chi_{j}^{s}\right]\left|\varphi\left(\theta_{n}^{j}\right)\right| d x+\varepsilon_{8}(n, j) .
\end{aligned}
$$


1040 Existence of solutions for elliptic equations

Combining this inequality with (3.22) and (3.39), we obtain

$$
\begin{aligned}
& \int_{\Omega}\left[a\left(x, T_{k}\left(u_{n}\right), \nabla T_{k}\left(u_{n}\right)\right)-a\left(x, T_{k}\left(u_{n}\right), \nabla T_{k}\left(v_{j}\right) \chi_{j}^{s}\right)\right]\left[\nabla T_{k}\left(u_{n}\right)-\nabla T_{k}\left(v_{j}\right) \chi_{j}^{s}\right] \\
& \quad \times\left[\varphi^{\prime}\left(\theta_{n}^{j}\right)-\frac{b(k)}{\alpha}\left|\varphi\left(\theta_{n}^{j}\right)\right|\right] d x \leq \varepsilon_{9}(n, j)+\int_{\Omega \backslash \Omega_{s}} h_{k} \nabla T_{k}(u) d x .
\end{aligned}
$$

Consequently,

$$
\begin{aligned}
& \int_{\Omega}\left[a\left(x, T_{k}\left(u_{n}\right), \nabla T_{k}\left(u_{n}\right)\right)-a\left(x, T_{k}\left(u_{n}\right), \nabla T_{k}\left(v_{j}\right) \chi_{j}^{s}\right)\right]\left[\nabla T_{k}\left(u_{n}\right)-\nabla T_{k}\left(v_{j}\right) \chi_{j}^{s}\right] d x \\
& \quad \leq 2 \varepsilon_{9}(n, j)+2 \int_{\Omega \backslash \Omega_{s}} h_{k} \nabla T_{k}(u) d x .
\end{aligned}
$$

On the other hand,

$$
\begin{aligned}
\int_{\Omega}[a(x, & \left.\left.T_{k}\left(u_{n}\right), \nabla T_{k}\left(u_{n}\right)\right)-a\left(x, T_{k}\left(u_{n}\right), \nabla T_{k}(u) \chi^{s}\right)\right]\left[\nabla T_{k}\left(u_{n}\right)-\nabla T_{k}(u) \chi^{s}\right] d x \\
= & \int_{\Omega}\left[a\left(x, T_{k}\left(u_{n}\right), \nabla T_{k}\left(u_{n}\right)\right)-a\left(x, T_{k}\left(u_{n}\right), \nabla T_{k}\left(v_{j}\right) \chi_{j}^{s}\right)\right]\left[\nabla T_{k}\left(u_{n}\right)-\nabla T_{k}\left(v_{j}\right) \chi_{j}^{s}\right] d x \\
& +\int_{\Omega} a\left(x, T_{k}\left(u_{n}\right), \nabla T_{k}\left(u_{n}\right)\right)\left[\nabla T_{k}\left(v_{j}\right) \chi_{j}^{s}-\nabla T_{k}(u) \chi^{s}\right] d x \\
& -\int_{\Omega} a\left(x, T_{k}\left(u_{n}\right), \nabla T_{k}(u) \chi^{s}\right)\left[\nabla T_{k}\left(u_{n}\right)-\nabla T_{k}(u) \chi^{s}\right] d x \\
& +\int_{\Omega} a\left(x, T_{k}\left(u_{n}\right), \nabla T_{k}\left(v_{j}\right) \chi_{j}^{s}\right)\left[\nabla T_{k}\left(u_{n}\right)-\nabla T_{k}\left(v_{j}\right) \chi_{j}^{s}\right] d x .
\end{aligned}
$$

We will pass to the limit in $n$ and in $j$ in the last three terms on the right-hand side of the above equality. Similar tools as in (3.24) and (3.41) give

$$
\begin{array}{r}
\int_{\Omega} a\left(x, T_{k}\left(u_{n}\right), \nabla T_{k}\left(u_{n}\right)\right)\left[\nabla T_{k}\left(v_{j}\right) \chi_{j}^{s}-\nabla T_{k}(u) \chi^{s}\right] d x=\varepsilon_{10}(n, j), \\
\int_{\Omega} a\left(x, T_{k}\left(u_{n}\right), \nabla T_{k}(u) \chi^{s}\right)\left[\nabla T_{k}\left(u_{n}\right)-\nabla T_{k}(u) \chi^{s}\right] d x=\varepsilon_{11}(n, j), \\
\int_{\Omega} a\left(x, T_{k}\left(u_{n}\right), \nabla T_{k}\left(v_{j}\right) \chi_{j}^{s}\right)\left[\nabla T_{k}\left(u_{n}\right)-\nabla T_{k}\left(v_{j}\right) \chi_{j}^{s}\right] d x=\varepsilon_{12}(n, j)
\end{array}
$$

which imply that

$$
\begin{aligned}
& \int_{\Omega}\left[a\left(x, T_{k}\left(u_{n}\right), \nabla T_{k}\left(u_{n}\right)\right)-a\left(x, T_{k}\left(u_{n}\right), \nabla T_{k}(u) \chi^{s}\right)\right]\left[\nabla T_{k}\left(u_{n}\right)-\nabla T_{k}(u) \chi^{s}\right] d x \\
& =\int_{\Omega}\left[a\left(x, T_{k}\left(u_{n}\right), \nabla T_{k}\left(u_{n}\right)\right)-a\left(x, T_{k}\left(u_{n}\right), \nabla T_{k}\left(v_{j}\right) \chi_{j}^{s}\right)\right]\left[\nabla T_{k}\left(u_{n}\right)-\nabla T_{k}\left(v_{j}\right) \chi_{j}^{s}\right] d x \\
& \quad+\varepsilon_{13}(n, j) .
\end{aligned}
$$


For $r \leq s$, one has

$$
\begin{aligned}
0 \leq & \int_{\Omega_{r}}\left[a\left(x, T_{k}\left(u_{n}\right), \nabla T_{k}\left(u_{n}\right)\right)-a\left(x, T_{k}\left(u_{n}\right), \nabla T_{k}(u)\right)\right]\left[\nabla T_{k}\left(u_{n}\right)-\nabla T_{k}(u)\right] d x \\
\leq & \int_{\Omega_{s}}\left[a\left(x, T_{k}\left(u_{n}\right), \nabla T_{k}\left(u_{n}\right)\right)-a\left(x, T_{k}\left(u_{n}\right), \nabla T_{k}(u)\right)\right]\left[\nabla T_{k}\left(u_{n}\right)-\nabla T_{k}(u)\right] d x \\
= & \int_{\Omega_{s}}\left[a\left(x, T_{k}\left(u_{n}\right), \nabla T_{k}\left(u_{n}\right)\right)-a\left(x, T_{k}\left(u_{n}\right), \nabla T_{k}(u) \chi^{s}\right)\right]\left[\nabla T_{k}\left(u_{n}\right)-\nabla T_{k}(u) \chi^{s}\right] d x \\
\leq & \int_{\Omega}\left[a\left(x, T_{k}\left(u_{n}\right), \nabla T_{k}\left(u_{n}\right)\right)-a\left(x, T_{k}\left(u_{n}\right), \nabla T_{k}(u) \chi^{s}\right)\right]\left[\nabla T_{k}\left(u_{n}\right)-\nabla T_{k}(u) \chi^{s}\right] d x \\
= & \int_{\Omega}\left[a\left(x, T_{k}\left(u_{n}\right), \nabla T_{k}\left(u_{n}\right)\right)-a\left(x, T_{k}\left(u_{n}\right), \nabla T_{k}\left(v_{j}\right) \chi_{j}^{s}\right)\right]\left[\nabla T_{k}\left(u_{n}\right)-\nabla T_{k}\left(v_{j}\right) \chi_{j}^{s}\right] d x \\
& +\varepsilon_{13}(n, j) \\
\leq & \varepsilon_{14}(n, j)+2 \int_{\Omega \backslash \Omega_{s}} h_{k} \nabla T_{k}(u) d x .
\end{aligned}
$$

This implies that, by passing at first to the limit sup over $n$ and next over $j$,

$$
\begin{aligned}
0 & \leq \underset{n \rightarrow \infty}{\limsup } \int_{\Omega_{r}}\left[a\left(x, T_{k}\left(u_{n}\right), \nabla T_{k}\left(u_{n}\right)\right)-a\left(x, T_{k}\left(u_{n}\right), \nabla T_{k}(u)\right)\right]\left[\nabla T_{k}\left(u_{n}\right)-\nabla T_{k}(u)\right] d x \\
& \leq 2 \int_{\Omega \backslash \Omega_{s}} h_{k} \nabla T_{k}(u) d x .
\end{aligned}
$$

Using the fact that $h_{k} \nabla T_{k}(u) \in L^{1}(\Omega)$ and letting $s \rightarrow \infty$, we get

$$
\int_{\Omega_{r}}\left[a\left(x, T_{k}\left(u_{n}\right), \nabla T_{k}\left(u_{n}\right)\right)-a\left(x, T_{k}\left(u_{n}\right), \nabla T_{k}(u)\right)\right]\left[\nabla T_{k}\left(u_{n}\right)-\nabla T_{k}(u)\right] d x \longrightarrow 0
$$

as $n \rightarrow \infty$.

As in [3], we deduce that there exists a subsequence still denoted by $u_{n}$ such that

$$
\nabla u_{n} \longrightarrow \nabla u \quad \text { a.e. in } \Omega,
$$

which implies that

$$
a\left(x, u_{n}, \nabla u_{n}\right) \rightarrow a(x, u, \nabla u) \text { weakly in }\left(L_{\bar{M}}(\Omega)\right)^{N} \quad \text { for } \sigma\left(\Pi L_{\bar{M}}, \Pi E_{M}\right) .
$$

Step 3 (modular convergence of the truncations). Going back to (3.46), we can write

$$
\begin{aligned}
\int_{\Omega} a\left(x, T_{k}\left(u_{n}\right), \nabla T_{k}\left(u_{n}\right)\right) \nabla T_{k}\left(u_{n}\right) d x \leq & \int_{\Omega} a\left(x, T_{k}\left(u_{n}\right), \nabla T_{k}\left(u_{n}\right)\right) \nabla T_{k}\left(v_{j}\right) \chi_{j}^{s} d x \\
& +\int_{\Omega} a\left(x, T_{k}\left(u_{n}\right), \nabla T_{k}\left(v_{j}\right) \chi_{j}^{s}\right) \\
& \times\left[\nabla T_{k}\left(u_{n}\right)-\nabla T_{k}\left(v_{j}\right) \chi_{j}^{s}\right] d x \\
& +2 \varepsilon_{9}(n, j)+2 \int_{\Omega \backslash \Omega_{s}} h_{k} \nabla T_{k}(u) d x,
\end{aligned}
$$


which implies, by using (3.50),

$$
\begin{aligned}
& \int_{\Omega} a\left(x, T_{k}\left(u_{n}\right), \nabla T_{k}\left(u_{n}\right)\right) \nabla T_{k}\left(u_{n}\right) d x \\
& \quad \leq \int_{\Omega} a\left(x, T_{k}\left(u_{n}\right), \nabla T_{k}\left(u_{n}\right)\right) \nabla T_{k}\left(v_{j}\right) \chi_{j}^{s} d x+\varepsilon_{15}(n, j)+2 \int_{\Omega \backslash \Omega_{s}} h_{k} \nabla T_{k}(u) d x .
\end{aligned}
$$

Passing to the limit sup over $n$ in both sides of this inequality yields

$$
\begin{aligned}
\limsup _{n \rightarrow \infty} \int_{\Omega} & a\left(x, T_{k}\left(u_{n}\right), \nabla T_{k}\left(u_{n}\right)\right) \nabla T_{k}\left(u_{n}\right) d x \\
& \leq \int_{\Omega} a\left(x, T_{k}(u), \nabla T_{k}(u)\right) \nabla T_{k}\left(v_{j}\right) \chi_{j}^{s} d x+\lim _{n \rightarrow \infty} \varepsilon_{15}(n, j)+2 \int_{\Omega \backslash \Omega_{s}} h_{k} \nabla T_{k}(u) d x,
\end{aligned}
$$

in which we can pass to the limit in $j$ to obtain

$$
\begin{aligned}
& \limsup _{n \rightarrow \infty} \int_{\Omega} a\left(x, T_{k}\left(u_{n}\right), \nabla T_{k}\left(u_{n}\right)\right) \nabla T_{k}\left(u_{n}\right) d x \\
& \quad \leq \int_{\Omega} a\left(x, T_{k}(u), \nabla T_{k}(u)\right) \nabla T_{k}(u) \chi^{s} d x+2 \int_{\Omega \backslash \Omega_{s}} h_{k} \nabla T_{k}(u) d x
\end{aligned}
$$

which gives, by letting $s \rightarrow \infty$,

$$
\limsup _{n \rightarrow \infty} \int_{\Omega} a\left(x, T_{k}\left(u_{n}\right), \nabla T_{k}\left(u_{n}\right)\right) \nabla T_{k}\left(u_{n}\right) d x \leq \int_{\Omega} a\left(x, T_{k}(u), \nabla T_{k}(u)\right) \nabla T_{k}(u) d x .
$$

On the other hand, we have, by using Fatou's lemma,

$$
\int_{\Omega} a\left(x, T_{k}(u), \nabla T_{k}(u)\right) \nabla T_{k}(u) d x \leq \liminf _{n \rightarrow \infty} \int_{\Omega} a\left(x, T_{k}\left(u_{n}\right), \nabla T_{k}\left(u_{n}\right)\right) \nabla T_{k}\left(u_{n}\right) d x,
$$

which implies that

$$
\int_{\Omega} a\left(x, T_{k}\left(u_{n}\right), \nabla T_{k}\left(u_{n}\right)\right) \nabla T_{k}\left(u_{n}\right) d x \longrightarrow \int_{\Omega} a\left(x, T_{k}(u), \nabla T_{k}(u)\right) \nabla T_{k}(u) d x \quad \text { as } n \longrightarrow \infty,
$$

and by using [4, Lemma 2.4], we conclude that

$$
a\left(x, T_{k}\left(u_{n}\right), \nabla T_{k}\left(u_{n}\right)\right) \nabla T_{k}\left(u_{n}\right) \longrightarrow a\left(x, T_{k}(u), \nabla T_{k}(u)\right) \nabla T_{k}(u) \quad \text { in } L^{1}(\Omega) .
$$

This implies, by using (3.4), that

$$
T_{k}\left(u_{n}\right) \longrightarrow T_{k}(u) \quad \text { in } W_{0}^{1} L_{M}(\Omega)
$$

for the modular convergence. 
Step 4 (equi-integrability of the nonlinearities and passage to the limit). We will prove that $g_{n}\left(x, u_{n}, \nabla u_{n}\right) \rightarrow g(x, u, \nabla u)$ strongly in $L^{1}(\Omega)$ by using Vitali's theorem.

Since $g_{n}\left(x, u_{n}, \nabla u_{n}\right) \rightarrow g(x, u, \nabla u)$ a.e. in $\Omega$, thanks to (3.55), it suffices to prove that $g_{n}\left(x, u_{n}, \nabla u_{n}\right)$ are uniformly equi-integrable in $\Omega$. Let $E \subset \Omega$ be a measurable subset of $\Omega$. We have, for any $m>0$,

$$
\begin{aligned}
\int_{E}\left|g_{n}\left(x, u_{n}, \nabla u_{n}\right)\right| d x= & \int_{E \cap\left\{\left|u_{n}\right| \leq m\right\}}\left|g_{n}\left(x, u_{n}, \nabla u_{n}\right)\right| d x+\int_{E \cap\left\{\left|u_{n}\right|>m\right\}}\left|g_{n}\left(x, u_{n}, \nabla u_{n}\right)\right| d x \\
\leq & b(m) \int_{E} a\left(x, T_{m}\left(u_{n}\right), \nabla T_{m}\left(u_{n}\right)\right) \nabla T_{m}\left(u_{n}\right) d x \\
& +b(m) \int_{E} c^{\prime}(x) d x+\frac{1}{m} \int_{\Omega} g_{n}\left(x, u_{n}, \nabla u_{n}\right) u_{n} d x .
\end{aligned}
$$

Standard arguments allow to deduce, using the strong convergence (3.64), that there exists $\mu>0$ such that

$$
|E|<\mu \Longrightarrow \int_{E}\left|g_{n}\left(x, u_{n}, \nabla u_{n}\right)\right| d x \leq \varepsilon, \quad \forall n,
$$

which shows that $g_{n}\left(x, u_{n}, \nabla u_{n}\right)$ are uniformly equi-integrable in $\Omega$ as required.

In order to pass to the limit, we have, by going back to approximate equations (3.13),

$$
\int_{\Omega} a\left(x, u_{n}, \nabla u_{n}\right) \nabla w d x+\int_{\Omega} g_{n}\left(x, u_{n}, \nabla u_{n}\right) w d x=\langle f, w\rangle
$$

for all $w \in \mathscr{D}(\Omega)$, in which, we can easily pass to the limit as $n \rightarrow \infty$ to get

$$
\int_{\Omega} a(x, u, \nabla u) \nabla w d x+\int_{\Omega} g(x, u, \nabla u) w d x=\langle f, w\rangle .
$$

Let now $v \in W_{0}^{1} L_{M}(\Omega) \cap L^{\infty}(\Omega)$. There exists $\left(w_{j}\right) \subset \mathscr{D}(\Omega)$ such that $\left\|w_{j}\right\|_{\infty, \Omega} \leq(N+$ 1) $\|v\|_{\infty, \Omega}$ for all $j \in \mathbb{N}$ and

$$
w_{j} \longrightarrow v
$$

for the modular convergence in $W_{0}^{1} L_{M}(\Omega)$. Taking $w=w_{j}$ in (3.69) and letting $j \rightarrow \infty$ yields

$$
\int_{\Omega} a(x, u, \nabla u) \nabla v d x+\int_{\Omega} g(x, u, \nabla u) v d x=\langle f, v\rangle
$$

By choosing $v=T_{k}(u)$ in the last equality, we get

$$
\int_{\Omega} a(x, u, \nabla u) \nabla T_{k}(u) d x+\int_{\Omega} g(x, u, \nabla u) T_{k}(u) d x=\left\langle f, T_{k}(u)\right\rangle .
$$

From (3.16), we deduce by Fatou's lemma that $g(x, u, \nabla u) u \in L^{1}(\Omega)$ and since $\mid g(x, u$, $\nabla u) T_{k}(u) \mid \leq g(x, u, \nabla u) u$ and $T_{k}(u) \rightarrow u$ in $W_{0}^{1} L_{M}(\Omega)$ for the modular convergence and 
a.e. in $\Omega$ as $k \rightarrow \infty$, it is easy to pass to the limit in both sides of (3.72) (by using Lebesgue theorem) to obtain

$$
\int_{\Omega} a(x, u, \nabla u) \nabla u d x+\int_{\Omega} g(x, u, \nabla u) u d x=\langle f, u\rangle .
$$

This completes the proof of Theorem 3.1.

Remark 3.4. If we replace, as in [5], (3.2) by the general growth condition

$$
|a(x, s, \xi)| \leq \bar{b}(|s|)\left(c(x)+\bar{M}^{-1} M(\gamma|\xi|)\right),
$$

where $\gamma>0, c \in E_{\bar{M}}(\Omega)$, and $\bar{b}: \mathbb{R}_{+} \rightarrow \mathbb{R}_{+}$is a continuous nondecreasing function, we prove the existence of solutions for the following problem:

$$
\begin{gathered}
u \in W_{0}^{1} L_{M}(\Omega), \quad g(x, u, \nabla u) \in L^{1}(\Omega), \quad g(x, u, \nabla u) u \in L^{1}(\Omega), \\
\left\langle A(u), T_{k}(u-v)\right\rangle+\int_{\Omega} g(x, u, \nabla u) T_{k}(u-v) d x \leq\left\langle f, T_{k}(u-v)\right\rangle \\
\forall v \in W_{0}^{1} L_{M}(\Omega) \cap L^{\infty}(\Omega) .
\end{gathered}
$$

Indeed, we consider the following approximate problems:

$$
\begin{gathered}
u_{n} \in W_{0}^{1} L_{M}(\Omega), \\
-\operatorname{div} a\left(x, T_{n}\left(u_{n}\right), \nabla u_{n}\right)+g_{n}\left(x, u_{n}, \nabla u_{n}\right)=f \quad \text { in } \Omega,
\end{gathered}
$$

and we conclude by adapting the same steps.

As an application of this result, we can treat the following model equations:

$$
-\operatorname{div}\left((1+|u|)^{m} \frac{\exp (|\nabla u|)-1}{|\nabla u|^{2}} \nabla u\right)+u \cos ^{2} u \exp (|\nabla u|)=f, \quad m \geq 0 .
$$

Remark that the solutions of (3.77) belong to $L^{\infty}(\Omega)$ so that (3.77) holds in the distributional sense.

\section{References}

[1] R. A. Adams, Sobolev Spaces, Academic Press, New York, 1975.

[2] A. Benkirane, Approximations de type Hedberg dans les espaces $W^{m} L \log L(\Omega)$ et applications [Hedberg-type approximations in the spaces $W^{m} L \log L(\Omega)$ and applications], Ann. Fac. Sci. Toulouse Math. (5) 11 (1990), no. 2, 67-78 (French).

[3] A. Benkirane and A. Elmahi, Almost everywhere convergence of the gradients of solutions to elliptic equations in Orlicz spaces and application, Nonlinear Anal. 28 (1997), no. 11, 1769-1784.

[4] An existence theorem for a strongly nonlinear elliptic problem in Orlicz spaces, Nonlinear Anal. Ser. A: Theory Methods 36 (1999), no. 1, 11-24.

[5] A. Benkirane, A. Elmahi, and D. Meskine, An existence theorem for a class of elliptic problems in $L^{1}$, Appl. Math. (Warsaw) 29 (2002), no. 4, 439-457.

[6] A. Benkirane and J.-P. Gossez, An approximation theorem in higher order Orlicz-Sobolev spaces and applications, Studia Math. 92 (1989), no. 3, 231-255. 
[7] A. Bensoussan, L. Boccardo, and F. Murat, On a nonlinear partial differential equation having natural growth terms and unbounded solution, Ann. Inst. H. Poincaré Anal. Non Linéaire 5 (1988), no. 4, 347-364.

[8] A. Elmahi, Existence results for strongly nonlinear elliptic unilateral problems in Orlicz spaces, to appear in Math-Recherche \& Applications.

[9] J.-P. Gossez, Nonlinear elliptic boundary value problems for equations with rapidly (or slowly) increasing coefficients, Trans. Amer. Math. Soc. 190 (1974), 163-205.

[10] Surjectivity results for pseudo-monotone mappings in complementary systems, J. Math. Anal. Appl. 53 (1976), no. 3, 484-494.

[11] Some approximation properties in Orlicz-Sobolev spaces, Studia Math. 74 (1982), no. 1, $17-24$.

[12] _ A strongly nonlinear elliptic problem in Orlicz-Sobolev spaces, Nonlinear Functional Analysis and Its Applications, Part 1 (Berkeley, Calif., 1983), Proc. Sympos. Pure Math., vol. 45, American Mathematical Society, Rhode Island, 1986, pp. 455-462.

[13] J.-P. Gossez and V. Mustonen, Variational inequalities in Orlicz-Sobolev spaces, Nonlinear Anal. 11 (1987), no. 3, 379-392.

[14] M. A. Krasnosel'skiǔ and Ja. B. Rutickiǔ, Convex Functions and Orlicz Spaces, P. Noordhoff, Groningen, 1961, translated from the first Russian edition by Leo F. Boron.

[15] A. Kufner, O. John, and S. Fučík, Function Spaces, Academia, Prague, 1977.

A. Elmahi: Centre Pédagogique Régional, B.P. 49, Fès 30000, Morocco

E-mail address: elmahi_abdelhak@yahoo.fr

D. Meskine: Département de Mathématiques et Informatique, Faculté des Sciences Dhar Mahraz, B.P. 1796, Atlas, Fès 30000, Morocco

E-mail address: meskinedriss@hotmail.com 


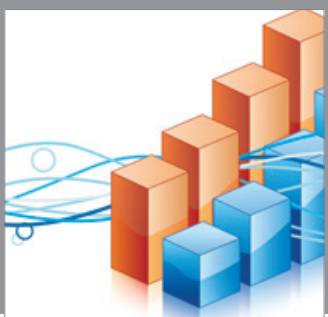

Advances in

Operations Research

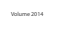

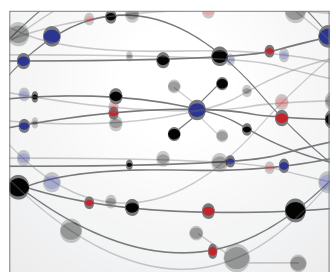

\section{The Scientific} World Journal
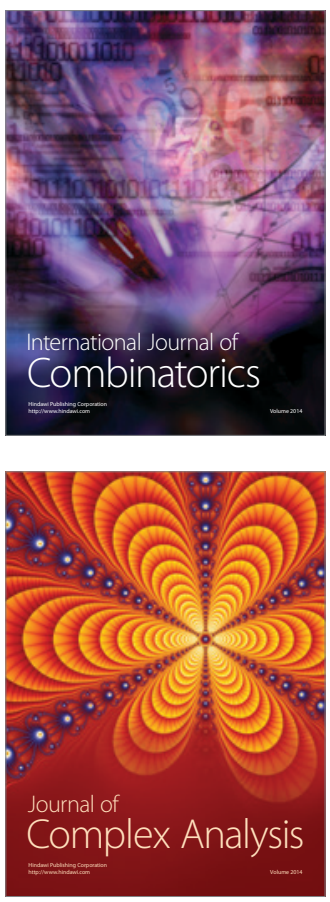

International Journal of

Mathematics and

Mathematical

Sciences
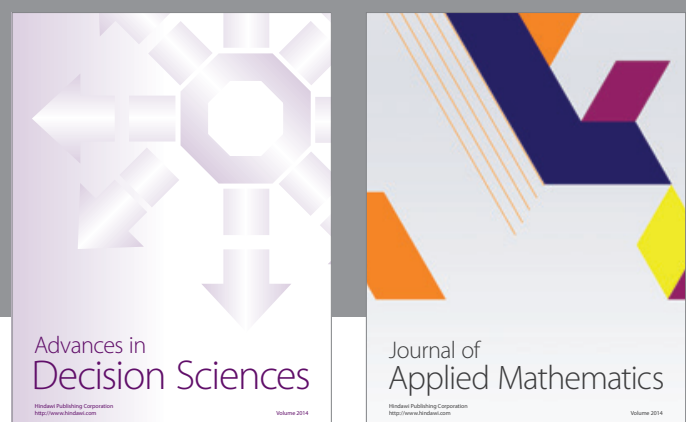

Journal of

Applied Mathematics
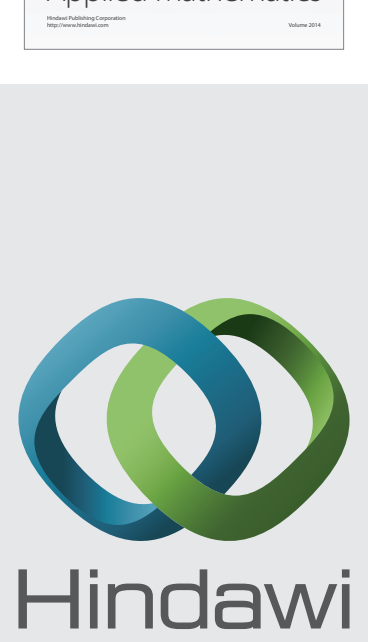

Submit your manuscripts at http://www.hindawi.com
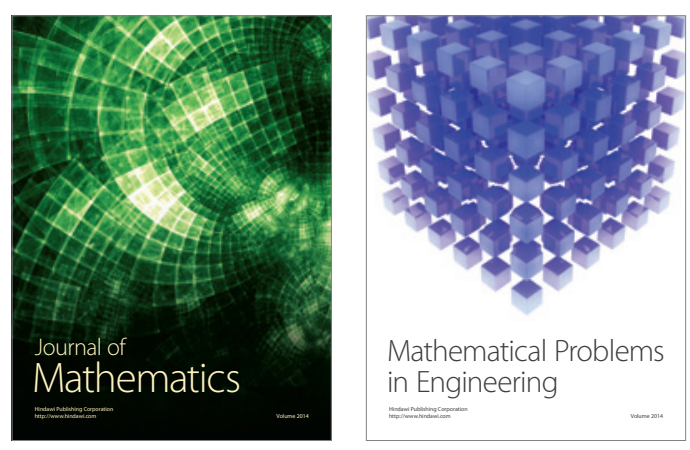

Mathematical Problems in Engineering
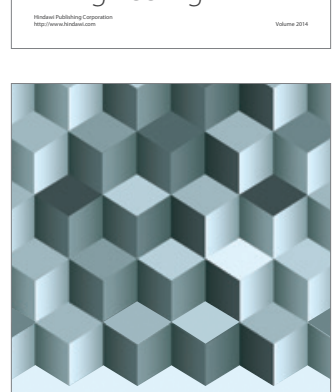

Journal of

Function Spaces
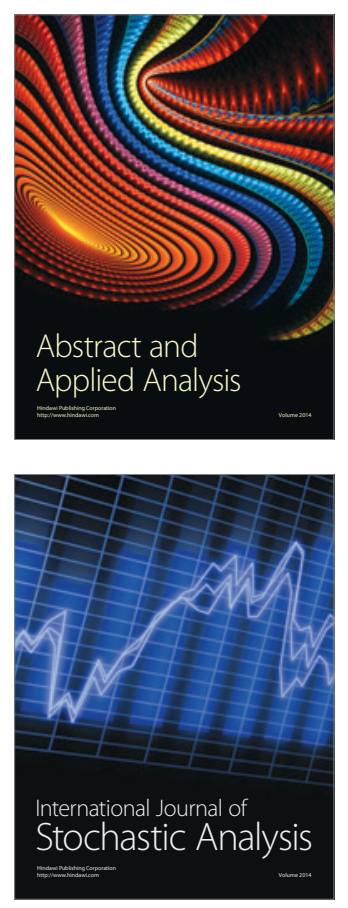

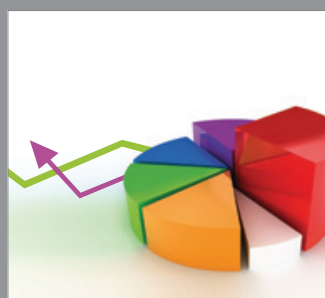

ournal of

Probability and Statistics

Promensencen
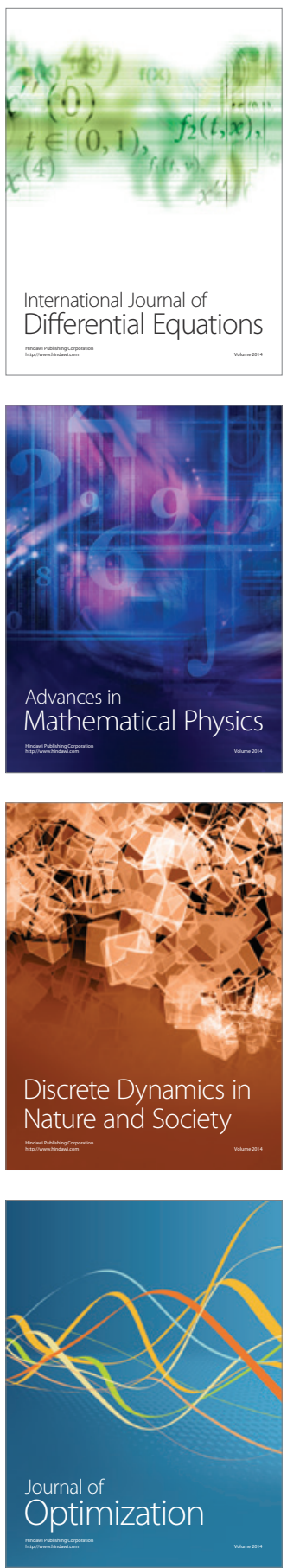